\title{
PHASE CALIBRATION OF THE PROPOSED MILLIMETER
}

\author{
ARRAY \\ M. A. HOLDAWAY \\ National Radio Astronomy Observatory, ${ }^{*}$ Socorro, New Mexico, 87801
}

January 8, 1993

\section{Introduction}

The Millimeter Array (MMA) is proposed to operate on baselines of up to $3 \mathrm{~km}$ at frequencies up to $350 \mathrm{GHz}$. Since no operating interferometer can indicate the expected phase stability on such baselines on high, dry sites, NRAO has undertaken a site survey, measuring the opacity and estimating the phase stability on baselines ranging from about $100 \mathrm{~m}$ to $5 \mathrm{~km}$ with a tipping radiometer. Radiometers have sampled the atmospheres at South Baldy, NM, and Springerville, AZ, and NRAO has recently started taking data at the 12,200 foot VLBA site on Mauna Kea.

\section{Estimating the Phase Structure Function}

Every five hours, the survey radiometer looked at the zenith for one hour, recording the emission at $225 \mathrm{GHz}$ from atmospheric water vapor. Assuming that inhomogeneously distributed water vapor dominates interferometric phase errors, the fluctuations recorded by the radiometer lead to the phase structure function (Holdaway, 1991). The most stable atmospheres above the Springerville and South Baldy sites are characterized by a single power law phase structure function:

$$
D_{\phi}(\rho)=\left(8.3 A S D_{56} \rho^{0.54}\right)^{2},
$$

where $\rho$ is the baseline in meters, $A S D_{56}$ is the Allan standard deviation of the radiometer fluctuations at $56 \mathrm{~s}$ averaging time, which varies from $0.03 \mathrm{~K}$ to $0.30 \mathrm{~K}$ for the best half of the winter months on the potential MMA sites, and $L_{\phi}$ is in units of degrees squared at $230 \mathrm{GHz}$. More commonly the phase structure function on the continental sites is a two part power law, consistent with Kolmogorov turbulence:

$$
\begin{array}{ll}
D_{\phi}(\rho)=\left(4 A S D_{56} \rho^{0.76}\right)^{2} & \rho<150 m \\
D_{\phi}(\rho)=\left(28 A S D_{56} \rho^{0.36}\right)^{2} & \rho>150 m .
\end{array}
$$

\section{Estimating Residual Calibration Errors}

Since the calibrator and the target source are observed through different paths in the atmosphere, and since the atmosphere is traveling with velocity $v$ across the array, errors will be made applying the calibrator phase to the target source.

* Associated Universities Inc. operates the National Radio Astronomy Observatory under National Science Foundation Cooperative Agreement No. AST-8814515 
Assuming a thin phase screen, the residual phase error after calibration will be independent of baseline for long baselines and is given by

$$
\sigma_{\phi}=\sqrt{2 D_{\phi}(v t+d)}
$$

where $D_{\phi}$ is the phase structure function, $t$ is the time required for the calibrator/target source observation cycle, and $d$ is the distance between the lines of site to the calibrator and the target source in the thin phase screen. Typical winds aloft on the potential MMA sites are about $12 \mathrm{~m} / \mathrm{s}$ during the winter months (Schwab, 1992).

An interferometer's phase is usually calibrated by observing a nearby point source on timescales of tens of minutes. Equation 3 indicates the rms phase on $3 \mathrm{~km}$ baselines will usually be more than a radian if calibration is performed only once every 10 minutes. The more frequently the array can be calibrated, the more often the array can be used on long baselines at high frequencies. To determine how frequently the array should be calibrated, we refer to Equation 4 . Since $D_{\phi}$ is a monotonically increasing function, we simply minimize the quantity $v t+d$ as a function of calibrator strength, slew rate, and array setup time and sensitivity for any particular calibration method. This optimization is carried out for several calibration techniques in Holdaway (1992a, 1992b). The MMA will be sensitive enough to get accurate phases on a $0.20 \mathrm{Jy}$ calibrator in under $3 \mathrm{~s}$, and source counts of flat spectrum quasars (Kühr, 1979) indicate that there will usually be a $0.20 \mathrm{Jy}$ calibrator within $2^{\circ}$ of the target source. Hence, it should be possible for the MMA to perform phase calibration with a cycle time of $10 \mathrm{~s}$ or less.

We are considering some novel phase calibration schemes: determining the atmospheric phase from total power fluctuations, frequent beam switching onto a nearby calibrator, and spatially pairing the antennas in the $3 \mathrm{~km}$ array such that one antenna is observing the calibrator while the other antenna is observing the target source. Any of these techniques will greatly increase the amount of time which the MMA will be able to observe on $3 \mathrm{~km}$ baselines at high frequencies. See Holdaway (1992b) for estimates of the $3 \mathrm{~km}$ array's usability with each of these calibration schemes.

\section{Preliminary Results From Mauna Kea}

Because the tipper data from Springerville suggests that the phase stability may be poor at high frequencies on long baselines, NRAO has begun looking at Mauna Kea in earnest. A tipping radiometer has been operating near the summit of Mauna Kea at the CSO for three years, but the highest site which could accommodate a $3 \mathrm{~km} \mathrm{MMA}$ is at 12,200 feet just north of the VLBA antenna. The phase stability at 12,200 feet may be much worse than at the summit because the inversion layer reaches the lower site more often and the summit may inject turbulent energy into the atmosphere. To study the phase stability and opacity at the 12,200 foot site, a second tipping radiometer began operation at the VLBA antenna in December, 1992.

Preliminary results from December of 1992 indicate: 
- The very best conditions occur equally frequently at the summit and the lower site.

- $\quad$ During times of good stability, the sky $T_{B}$ fluctuations are typically $25 \%$ higher at the VLBA site.

- There have been no instances of good seeing at the CSO site and poor seeing at the VLBA site.

- The temporal sky $T_{B}$ structure function often flattens to a 0.33 power law between 30 and $300 \mathrm{~s}$.

If we take the sky brightness temperature fluctuation data at face value, the month of December at Mauna Kea is comparable to the months of best atmospheric stability on South Baldy. However, there are several important factors which complicate the comparison between South Baldy and Mauna Kea:

- December is the month of worst seeing on Mauna Kea, but one of the best on South Baldy.

- Because the sites are at different elevations, the pressure broadening will be different at the two sites, affecting the conversion of $T_{B}$ fluctuations into water vapor column density, and hence phase, fluctuations.

- The differing atmospheric temperatures will also affect conversion of $T_{B}$ fluctuations into phase fluctuations.

- The atmospheric velocity at the South Baldy site is about $12 \mathrm{~m} / \mathrm{s}$ during excellent seeing, while the velocity above Mauna Kea is about $5 \mathrm{~m} / \mathrm{s}$. The temporal phase structure function is related to the spatial phase structure function as

$$
D_{\phi}(t)=D_{\phi}\left(\rho / v_{a t m}\right) \text {. }
$$

The structure function evaluated at $\rho=v t+d$ is the relevant quantity to study when external calibration is performed:

$$
D_{\phi}(t)=D_{\phi}\left(\left(v_{a t m} t+d\right) / v_{a t m}\right) \text {. }
$$

This will affect how well different calibration strategies will work at the two sites.

Obviously, we cannot make a legitimate comparison between the Mauna Kea and South Baldy atmospheric phase stabilities until an entire year of data has been collected from Mauna Kea and the differences in the physical conditions at the sites have been correctly accounted for. However, the preliminary data indicate that the 12,200 foot site on Mauna Kea may provide excellent phase stability.

\section{References}

Holdaway, M.A., 1991, A Millimeter Wavelength Phase Stability Analysis of the South Baldy andSpringerville Sites, MMA Memorandum 68, NRAO: Socorro, NM

Holdaway, M.A., 1992a, Possible Phase Calibration Schemes for the MMA, MMA Memorandum 84, NRAO: Socorro, NM

Holdaway, M.A., 1992b, Paired Antenna Phase Calibration: Residual Phase Errors and Configuration Study, MMA Memorandum 88, NRAO: Socorro, NM

Kühr, H., et al, 1979, MPIFR Preprint No. 55.

Schwab, F.R., 1992, Lower Tropospheric Wind Speed Statistics at Albuquerque, NM, Winslow, $A Z$, and Hilo, $H A$, MMA Memorandum 75, NRAO: Socorro, NM 


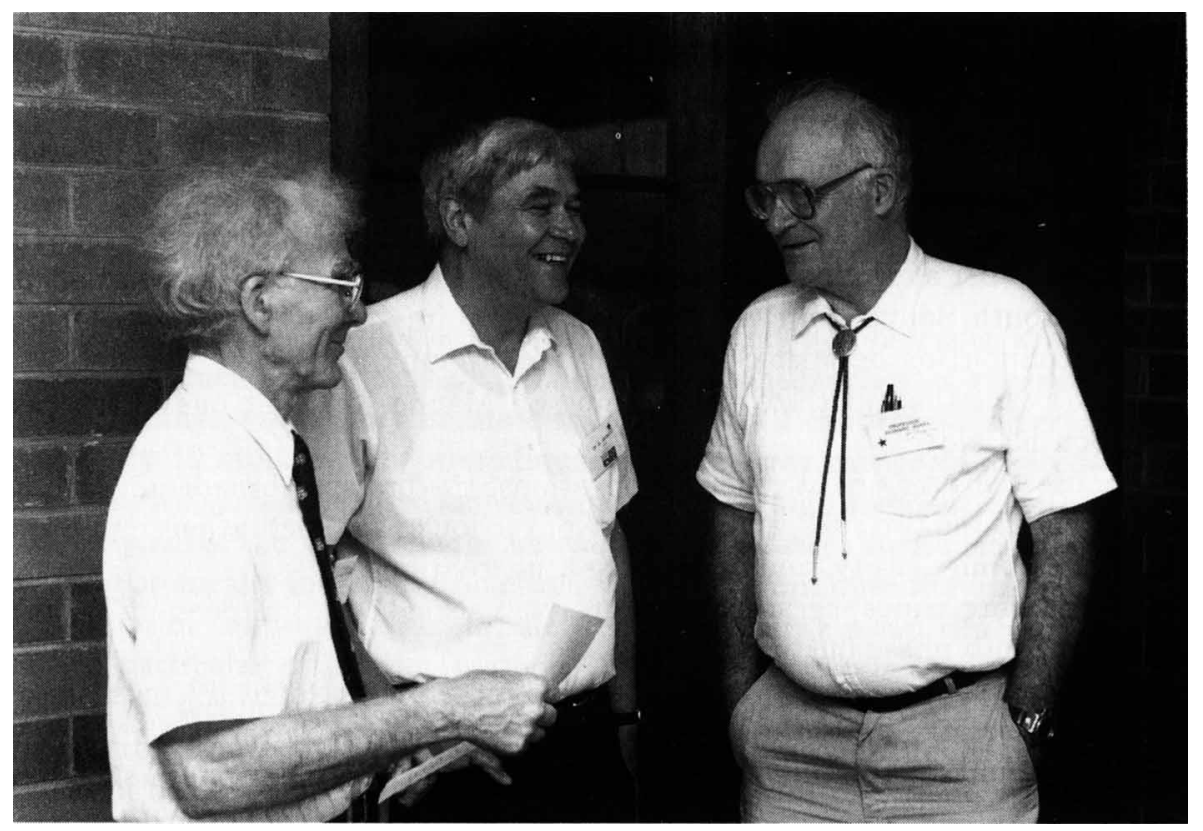

